\title{
Optimization Algorithm for Spectrum Sensing Delay Time in Cognitive Radio Networks Using Decoding Forward Relay
}

\author{
Kaili Xia, Xianyang Jiang*, Yingbiao Yao, and Xianghong Tang \\ School of Communication Engineering, Hangzhou Dianzi University \\ Hangzhou, Zhejiang 310018, P.R. China \\ [e-mail: xkl19920701@163.com] \\ [e-mail: jiangxy@hdu.edu.cn] \\ *Corresponding author: Xianyang Jiang
}

Received January 28, 2018; revised April 21, 2019; accepted November 22, 2019; published March 31, 2020

\begin{abstract}
Using decode-and-forward relaying in the cognitive radio networks, the spectrum efficiency can improve furthermore. The optimization algorithm of the spectrum sensing estimation time is presented for the cognitive relay networks in this paper. The longer sensing time will bring two aspects of the consequences. On the one hand, the channel parameters are estimated more accurate so as to reduce the interferences to the authorized users and to improve the throughput of the cognitive users. On the other hand, it shortens the transmission time so as to decease the system throughput. In this time, it exists an optimal sensing time to maximize the throughput. The channel state information of the sub-bands is considered as the exponentially distributed, so a stochastic programming method is proposed to optimize the sensing time for the cognitive relay networks. The computer simulation results using the Matlab software show that the algorithm is effective, which has a certain engineering application value.
\end{abstract}

Keywords: Cognitive relay networks, decode-and-forward relay, spectrum sensing, random programming.

This research was supported by a research from the Zhejiang Province Science Foundation for Public Welfare under Grant LGG19F020014, and supported by the Program of Zhejiang Provincial Natural Science Foundation of China under Grant No. LY19F010011. 


\section{Introduction}

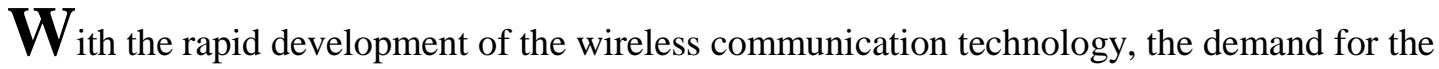
spectrum resource has become increasingly tense. To solve this contradiction, the current spectrum management method must be improved. The Cognitive Radio (CR) is one of the most promising techniques to solve the spectrum scarcity. Some existing researches have focused on improving the spectrum efficiency and the power allocation optimization of the cognitive radio system [1]. On the other hand, the cooperative relaying technology is the key technology to improve the spectrum utilization, which has been shown effective to improve the capacity, promote the transmission reliability and increase the coverage of the wireless communication networks. The combination of the cognitive radio and the cooperative relaying technology can effectively improve the performance of the wireless network system and obtain higher spectrum utilization [2]. The cognitive relay networks include two basic strategies, the amplify-and-forward (AF) and the decode-and-forward (DF). In the DF scheme, the relay demodulates the received signal before retransmission, whereas in the AF scheme, the relay just amplifies the received signal only. In this paper, the DF relaying strategy is analysed.

The cognitive user generally applies the listen-before-transmit method to sense the spectrum of the primary user channel at the beginning of each data transmission frame. If the detected channel of the primary user is idle, the cognitive user transmits the data using the channel [3]. G. Ozcan proposed a cooperative detection algorithm of the cognitive radio spectrum based on the amplification and relaying technology [4]. Q. Cui analyzed the influence of the transmission time and power on the energy efficiency of the relay communication under the DF protocol [5]. X. Jiang introduced a power allocation optimization algorithm into the CRNs, proposed an interference evaluation strategy to use the different spectrum and power allocation methods while the secondary users are located in the different service regions of the primary users [6].

In this paper, the cognitive relay network model is used to apply the decode-and-forward protocols, which is put forward by Sendonaris [7][8], and also referred to as the Regenerative Relay (RR) processing mode [9][10]. The main idea is adopted that the information is transmitted from the source to the relay and the destination in the first time slot, then, the relay decode the received information, recode and forward to the destination in the second time slot. The destination decode the two parts of information and combine them to get the original information. In paper [11], Liu pointed out that the performance of the cognitive radio networks is influenced by the sensing time, and longer sensing time will improve the system performance, but also reduce the inter-channel data transmission time. Therefore, appropriately chosing the sensing time is very necessary to improve the performance of the cognitive relay networks. So, in this paper, it is necessary to optimize the spectral sensing time of the cognitive relay networks for the DF scheme.

The main contributions of this paper are summarised as follows: (1) A time-delay model is presented for the spectrum sensing process in the cognitive relay networks using the DF protocol; (2) A stochastic programming method is proposed to solve the time-delay model in 
the cognitive relay networks. The rest of this paper is organised as follows. The system model is presented in Section 2. Section 3 proposes the optimization method of the spectrum sensing time in the cognitive DF-relay networks, then the stochastic programming model is introduced to solve the optimization problem. The numerical results and the CRNs' performances are discussed in Section 4. Finally, Section 5 presets the conclusions of this study.

\section{System Model}

The cognitive relay system is composed of a Primary User (PU) and a Seconary User (SU), in which the SU is made up of a Cognitive Source (CS), a Cognitive Relay (CR) and a Cognitive Destination (CD), as shown in Fig. 1. $G_{r p}, G_{s p}$ are the channel gains from the CR and the CS to the PU respectively. $G_{s d}, G_{s r}, G_{r d}$ are denoted the channel gains between the CS, CR and $\mathrm{CD}$, as $C S \rightarrow C D, C S \rightarrow C R, C R \rightarrow C D$

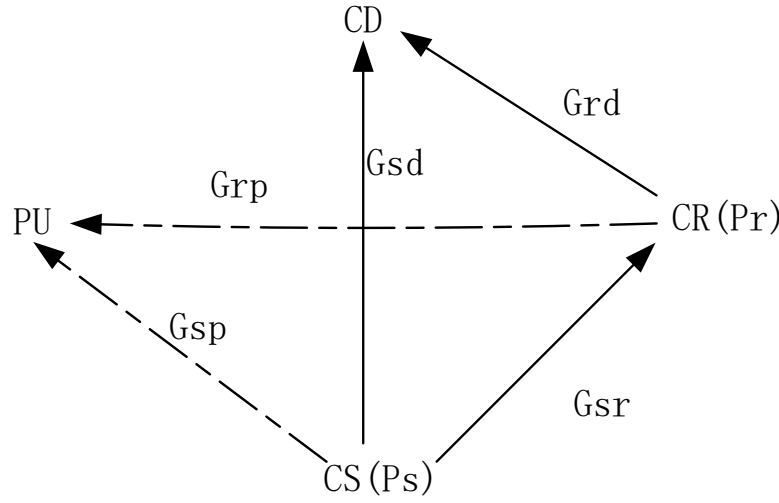

(a)

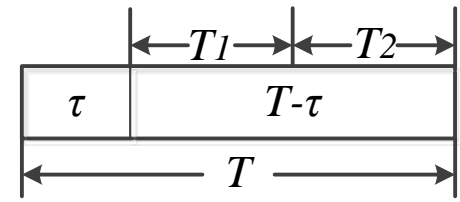

(b)

Fig. 1. DF cognitive relay network model.

In Fig. 1 (a), the signal transmission from the CS to the CD is divided into two periods. In the $T_{1}$ period, the CS sends a signal, which is received by the CR and the CD; In the $T_{2}$ period, the CR transmits the signal received from the CS and decoded using the DF scheme, then, the CD receives the signal and merges it with the received signal at the $T_{1}$ period using the maximum ratio combination. The CS also receives the signal transmitted from the CR for the channel estimation. In Fig. 1(b), the total time of one data frame is denoted as $T$, which contains the duration $\tau$ for the spectrum sensing and channel estimation. The remaining time $T-\tau$ is used for information transmission, which is devided into two equal periods, $T_{1}+T_{2}$, as shown in Fig. 1.

In general, it takes longer in the spectrum sensing, the channel estimation results are more accurate, and there are less interferences to the PU produced by the SU. Moreover, the SU's power allocation algorithm is more reasonable, the data transmission rate is even higher. However, the longer spectrum sensing time can lead to excessive resource consumption of the data transmission, resulting in lower system throughput. Therefore, the spectrum sensing time 
delay $\tau$ is an optimized parameter, which can balance the effects of the above mentioned both aspects to achieve the highest data transmission rate.

\section{Optimization Algorithm of Spectrum Sensing Time Delay}

\section{1 miss detection probability and false alarm probability}

Here, the energy detection method is applied in the spectrum sensing. The transmission signal is a complex-valued MPSK modulation signal with an independent and symmetric cyclic symmetric complex Gaussian white noise. The miss detection probability $p_{m d}$ and the false alarm probability $p_{f a}$ can be expressed as

$$
\begin{aligned}
& p_{m d}(\tau)=1-Q\left(\left(\frac{\lambda}{N_{0}}-\eta-1\right) \sqrt{\frac{\tau f_{s}}{2 \eta+1}}\right) \\
& p_{f a}(\tau)=Q\left(\left(\frac{\lambda}{N_{0}}-1\right) \sqrt{\tau f_{s}}\right)
\end{aligned}
$$

Where,

$$
Q(x)=\frac{1}{\sqrt{2 \pi}} \int_{x}^{\infty} \exp \left(-\frac{u^{2}}{2}\right) d u
$$

$\lambda$ is the energy threshold which determines whether the PU is idle or not, $\eta$ is the signal-to-noise ratio received by the CS from the PU, $f_{s}$ is the sampling frequency of the CS, $N_{0}$ is the noise power.

\subsection{Model establishment}

Compared with the AF protocol, the DF relay can avoid the noise being amplified and improve the signal quality. The throughput of the cognitive relay network system under the DF protocol can be expressed as,

$$
R\left(\tau, P_{s}, P_{r}, \xi\right)=\frac{T-\tau}{T} \psi(\xi) \min \left(\log _{2}\left(1+\frac{\gamma(\xi)}{N_{0}}\right), \log _{2}\left(1+\frac{\beta(\xi)}{N_{0}}+\frac{\rho(\xi)}{N_{0}}\right)\right)
$$

where $\xi=\left\lfloor G_{s d}, G_{s r}, G_{r d}, G_{r p}, G_{s p}, \chi, \tilde{\chi}\right\rfloor$ is the vector of the random variables, $N_{0}$ is the noise power. $\chi$ is the PU channel activity state judgment variable ( $\chi \in\{O$ :Occupied, $V:$ Vacant $\}$ ). $\tilde{\chi}$ represents the cognitive source spectrum sensing decision $\tilde{\chi} \in\{\mathrm{O}, \mathrm{V}\}$.

$$
\tilde{\chi}= \begin{cases}V & \text { CS or CR detects channel vacant } \\ O & \text { CS or CR detects channel occupied }\end{cases}
$$

In Eq. 4,

$$
\gamma(\xi)=P_{s} G_{s d}, \quad \beta(\xi)=P_{s} G_{s r}, \quad \rho(\xi)=P_{r} G_{r d} .
$$

where $P_{s}$ is the transmission power of the cognitive source CS, $P_{r}$ is the transmission power of the cognitive relay CR. $\psi(\xi)$ is the indicator function, indicating whether the CS transmits 
data or not according to the detection results. If the CS transmits the signal, $\psi(\xi)=1$, otherwise, $\psi(\xi)=0$.

$$
\psi(\xi)= \begin{cases}1 & \tilde{\chi}=V \\ 0 & \tilde{\chi}=O\end{cases}
$$

If $v=\operatorname{Pr}(\chi=V)$ is denoted as the channel vacant probability, the probability quality function of $\psi(\xi)$ can be represented as the following equation,

$$
\operatorname{Pr}(\psi(\xi)=1)=\left[1-p_{f a}(\tau)\right] v+p_{m d}(\tau)(1-v)=p_{\psi 1}
$$

The optimization issues can be expressed as,

Subject to,

$$
\max _{\tau, P_{s}, P_{r}} R\left(\tau, P_{s}, P_{r}, \xi\right)
$$

$$
\begin{aligned}
& 0 \leq P_{s} \leq P_{s}^{m}, \\
& 0 \leq P_{r} \leq P_{r}^{m}, \\
& E\left[I_{s}\left(P_{s}, \xi\right)\right] \leq I_{s}^{a}, \\
& E\left[I_{r}\left(P_{r}, \xi\right)\right] \leq I_{r}^{a}, \\
& 0 \leq \tau \leq T .
\end{aligned}
$$

$P_{s}{ }^{m}$ is the maximum power constraint threshold for the cognitive source CS. $P_{r}{ }^{m}$ is the maximum power constraint threshold for the CR. In the case of the Rayleigh fading channel, the channel gain complies with the exponential distribution. In general, it is difficult to obtain all the channel state information (CSI), because the cooperation between the PU and the SU may bring a lot of overhead to the PU. So this paper assumes that only the average of the interference channel gain $G_{s p}, G_{r p}$ is known. Assume that $I_{s}{ }^{a}$ and $I_{r}{ }^{a}$ is the average interference constraint from the CS and the CR to the PU. $E\left[I_{s}\left(P_{s}, \xi\right)\right]$ is the mean value of the interference power of the CS on the PU, and $E\left[I_{r}\left(P_{r}, \xi\right)\right]$ is the mean value of the interference power of the CR on the PU.

$$
I_{s}\left(P_{s}, \xi\right)=G_{s p} \cdot P_{s} \cdot \vartheta(\chi, \tilde{\chi})
$$

where $\vartheta(\chi, \tilde{\chi})$ indicates whether the spectrum sensing result will interfere with the subband that the PU is occupying, that is, it equals to 1 while the actual subband is occupied but the result of the spectrum sensing is idle. That is,

$$
\vartheta(\chi, \tilde{\chi})= \begin{cases}1 & (\chi=O, \tilde{\chi}=V) \\ 0 & \text { others }\end{cases}
$$

The probabilistic mass function of $\vartheta(\chi, \tilde{\chi})$ can be expressed as,

$$
\operatorname{Pr}((\chi, \tilde{\chi})=1)=\operatorname{Pr}(\chi=\mathrm{O}, \tilde{\chi}=\mathrm{V})=\operatorname{Pr}(\tilde{\chi}=\mathrm{V} \mid \chi=\mathrm{O}) \operatorname{Pr}(\chi=\mathrm{O})=\mathrm{p}_{\mathrm{md}}(\tau) \mathrm{V}
$$

Similarly, the interference power of the CR to the PU can be denoted as,

$$
I_{r}\left(P_{r}, \xi\right)=G_{r p} \cdot P_{r} \cdot \vartheta(\chi, \tilde{\chi})
$$


As a reasonable assumption, the interference instruction function $\vartheta(\chi, \tilde{\chi})$ and the channel gain $G_{s p}, G_{r p}$ are not relevant. The tolerable average interference constraint can be expressed as,

$$
\begin{array}{ll}
E\left[I_{s}\left(P_{s}, \xi\right)\right]=E\left[P_{s} G_{s p}\right. & (\chi, \tilde{\chi})]=P_{s} E\left[G_{s p}(\chi, \tilde{\chi})\right]=P_{s} \cdot p_{m d} \cdot v \cdot \Omega_{s p} \leq I_{s}^{a} \\
E\left[I_{r}\left(P_{r}, \xi\right)\right]=E\left[P_{r} G_{r p}\right. & (\chi, \tilde{\chi})]=P_{r} E\left[G_{r p}(\chi, \tilde{\chi})\right]=P_{r} \cdot p_{m d} \cdot v \cdot \Omega_{r p} \leq I_{r}^{a}
\end{array}
$$

where $\Omega_{s p}$ are the mean value of the interference channel gain from the CS on the PU, and $\Omega_{r p}$ denotes the interference channel gain from the CR on the PU. It can be seen in Eq. 9-10 that the model is a complex optimization problem which can not be directly calculated. A stochastic programming method is proposed in the following section to solve this complicated problem.

\subsection{Stochastic Programming Model}

The random programming algorithm includes three basic steps.

Step 1. Solve the problem, $R(\tau, \xi)$,

$$
R(\tau, \xi)=\max _{P_{s}, P_{r}} R\left(\tau, P_{s}, P_{r}, \xi\right)
$$

Step 2. Solve the problem, $R(\tau)$,

$$
R(\tau)=E_{\xi}[R(\tau, \xi)]
$$

Step 3. Solve the problem, $\max _{\tau} R(\tau)$.

The average value of the interference channel gains $G_{s p}, G_{r p}$ are assumed to be known. The average transmission power of the CS and the CR can be expressed as,

$$
\begin{aligned}
& \bar{P}_{s}=\frac{I_{r}^{a}}{E\left[G_{s p}(\chi, \tilde{\chi})\right]}=\frac{I_{r}^{a}}{p_{m d}(\tau) \cdot v \cdot \Omega_{s p}} \\
& \bar{P}_{r}=\frac{I_{r}^{a}}{E\left[G_{r p}(\chi, \tilde{\chi})\right]}=\frac{I_{r}^{a}}{p_{m d}(\tau) \cdot v \cdot \Omega_{r p}}
\end{aligned}
$$

So the signal power received by the CD from the CS channel can be expressed indirectly as,

$$
\gamma(\xi)=\min \left(P_{m}^{s}, \bar{P}_{s}\right) G_{s d}
$$

Similarly, the signal power received from the CS to the CR channel, and the signal power received from the $\mathrm{CR}$ to the $\mathrm{CD}$ channel, are respectively denoted as following.

$$
\begin{aligned}
& \beta(\xi)=\min \left(P_{s}^{m}, \overline{P_{s}}\right) G_{s r} \\
& \rho(\xi)=\min \left(P_{r}^{m}, \bar{P}_{r}\right) G_{r d}
\end{aligned}
$$

It is assumed that the transmission channel gain $G_{s d}, G_{s r}$, and $G_{r d}$ are with the exponential distribution $\Omega_{s d}, \Omega_{s r}$, and $\Omega_{r d}$. According to the exponential distribution characteristic, the probability functions of $\gamma, \beta, \rho$ comply with the exponential distribution as shown in following. 


$$
\left\{\begin{array}{l}
\Omega_{\gamma}=\min \left(P_{s}^{m}, \overline{P_{s}}\right) \Omega_{s d} \\
\Omega_{\beta}=\min \left(P_{s}^{m}, \overline{P_{s}}\right) \Omega_{s r} \\
\Omega_{\rho}=\min \left(P_{r}^{m}, \bar{P}_{r}\right) \Omega_{r d}
\end{array}\right.
$$

Then the conditional probability density of $\gamma, \beta, \rho$ is the exponential distribution of $\Omega_{\gamma}, \Omega_{\beta}$, and $\Omega_{\rho}$.

$$
\left\{\begin{array}{l}
f_{\gamma \mid \tilde{\chi}=V}(x)=\frac{1}{\Omega_{\gamma}} e^{-\frac{x}{\Omega_{\gamma}}} \\
f_{\beta \mid \tilde{\chi}=V}(y)=\frac{1}{\Omega_{\beta}} e^{-\frac{x}{\Omega_{\beta}}} \\
f_{\rho \mid \tilde{\chi}=V}(z)=\frac{1}{\Omega_{\rho}} e^{-\frac{x}{\Omega_{\rho}}}
\end{array}\right.
$$

According to Eq. 4, $R(\tau)$ can be expressed as

$$
\begin{aligned}
R(\tau)= & E_{\xi}[R(\tau, \xi)] \\
= & \frac{T-\tau}{T} p_{\psi 1} \times\left\{\begin{array}{l}
E_{\xi}\left[\log _{2}\left(1+\frac{\gamma(\xi)}{N_{0}}\right) \mid \tilde{\chi}=V\right], \gamma(\xi) \leq \beta(\xi)+\rho(\xi) \\
E_{\xi}\left[\log _{2}\left(1+\frac{\beta(\xi)}{N_{0}}+\frac{\rho(\xi)}{N_{0}}\right) \mid \tilde{\chi}=V\right], \gamma(\xi)>\beta(\xi)+\rho(\xi)
\end{array}\right.
\end{aligned}
$$

In the case of a known average interference channel gain, the throughput of the first stage can be expressed as

$$
R(\tau)=\frac{T-\tau}{T} p_{\psi 1} \bar{R}
$$

where

$$
\begin{aligned}
\bar{R}= & \iiint_{\substack{x<y+z \\
x>0, y>0, z>0}} \log _{2}\left(1+\frac{x}{N_{0}}\right) f_{\gamma \mid \tilde{\chi}=V}(x) f_{\beta \mid \tilde{\chi}=V}(y) f_{\rho \mid \tilde{x}=V}(z) d x d y d z \\
& +\iiint_{\substack{x>y+z \\
x>0, y>0, z>0}} \log _{2}\left(1+\frac{y}{N_{0}}+\frac{z}{N_{0}}\right) f_{\gamma \mid \tilde{\chi}=V}(x) f_{\beta \mid \tilde{x}=V}(y) f_{\rho \mid \tilde{\chi}=V}(z) d x d y d z
\end{aligned}
$$

which can be calculated as following, 


$$
\begin{aligned}
& \bar{R}=\frac{\Omega_{\beta}}{\ln 2 \cdot\left(\Omega_{\rho}-\Omega_{\beta}\right)} e^{N_{0}\left(\frac{1}{\Omega_{\gamma}}+\frac{1}{\Omega_{\beta}}\right)} E i\left[-N_{0}\left(\frac{1}{\Omega_{\gamma}}+\frac{1}{\Omega_{\beta}}\right)\right] \\
& +A \cdot e^{N_{0}\left(\frac{1}{\Omega_{\gamma}}+\frac{1}{\Omega_{\rho}}\right)} E i\left[-N_{0}\left(\frac{1}{\Omega_{\gamma}}+\frac{1}{\Omega_{\rho}}\right)\right] \\
& -\frac{1}{\ln 2} e^{\frac{N_{0}}{\Omega_{\gamma}}} E i\left[-\frac{N_{0}}{\Omega_{\gamma}}\right]
\end{aligned}
$$

where

$$
A=\frac{\Omega_{\gamma}{ }^{2} \Omega_{\rho} \Omega_{\beta}-\Omega_{\gamma}{ }^{2} \Omega_{\rho}{ }^{2}+\Omega_{\gamma} \Omega_{\rho}{ }^{2}+2 \cdot \Omega_{\gamma}{ }^{2} \Omega_{\rho}+\Omega_{\beta} \Omega_{\rho}{ }^{2}-\Omega_{\beta}{ }^{2} \Omega_{\gamma}+\Omega_{\gamma} \Omega_{\rho} \Omega_{\beta}}{\ln 2 \cdot\left(\Omega_{\gamma}+\Omega_{\beta}\right) \cdot\left(\Omega_{\gamma}+\Omega_{\rho}\right) \cdot\left(\Omega_{\beta}-\Omega_{\rho}\right)}
$$

and $\operatorname{Ei}(x)=\int_{-\infty}^{x} e^{t} / t d t$. Then, the system throughput $R(\tau)$ is obtained as follows.

$$
\begin{array}{r}
R(\tau)=\frac{T-\tau}{T} p_{\psi 1} \times\left(\frac{\Omega_{\beta}}{\ln 2 \cdot\left(\Omega_{\rho}-\Omega_{\beta}\right)} e^{N_{0}\left(\frac{1}{\Omega_{\gamma}}+\frac{1}{\Omega_{\beta}}\right)} E \mathrm{i}\left[-N_{0}\left(\frac{1}{\Omega_{\gamma}}+\frac{1}{\Omega_{\beta}}\right)\right]\right. \\
\left.+A \cdot e^{N_{0}\left(\frac{1}{\Omega_{\gamma}}+\frac{1}{\Omega_{\rho}}\right)} E \mathrm{E}\left[-N_{0}\left(\frac{1}{\Omega_{\gamma}}+\frac{1}{\Omega_{\rho}}\right)\right]-\frac{1}{\ln 2} e^{\frac{N_{0}}{\Omega_{\gamma}}} E i\left[-\frac{N_{0}}{\Omega_{\gamma}}\right]\right)
\end{array}
$$

To solve the optimization function shown in Eq. 31 is very complex and difficult using the usual unconstrained optimization algorithm, because of the minimum function existing in Eq. 24. The Monte Carlo algorithm is used to obtain the maximum throughput.

\section{Experimental Results and Analysis}

The simulation parameters are set as shown in Table 1 [12].

Table 1. Simulation parameter setting.

\begin{tabular}{|c|c|}
\hline Parameters & Values \\
\hline$\lambda$ & 0.055 \\
\hline$N_{0}$ & $0.05 \mathrm{~mW}$ \\
\hline$\eta$ & $-6 d B$ \\
\hline$T$ & $5 \mathrm{~ms}$ \\
\hline$f_{s}$ & $10 \mathrm{~K}$ samples $/ \mathrm{s}$ \\
\hline$v$ & 0.7 \\
\hline
\end{tabular}

The communication system apply in the complex MPSK modulation with the parameters set in Table 1. The index distribution rate of each channel gain $\Omega_{s d}, \Omega_{s r}, \Omega_{r d}, \Omega_{r p}$, and $\Omega_{s p}$ are set 1 . 
$P_{m}{ }^{s}$ and $P_{m}{ }^{r}$ are set $-10 \mathrm{dBm}$. The communication networks are simulated using the MATLAB software to solve Eq. 31. The Monte Carlo method is used with 1000 times of the test number. The optimal time obtained as $0.615 \mathrm{~ms}$. The result is as shown in Figs. 2-5. the sensing time $\tau$ gets longer, the throughput firstly increases and reaches a maximum value, then starts to decrease. There is a maximum value of the throughput with the optimal solution of the sensing time $\tau_{0}$.

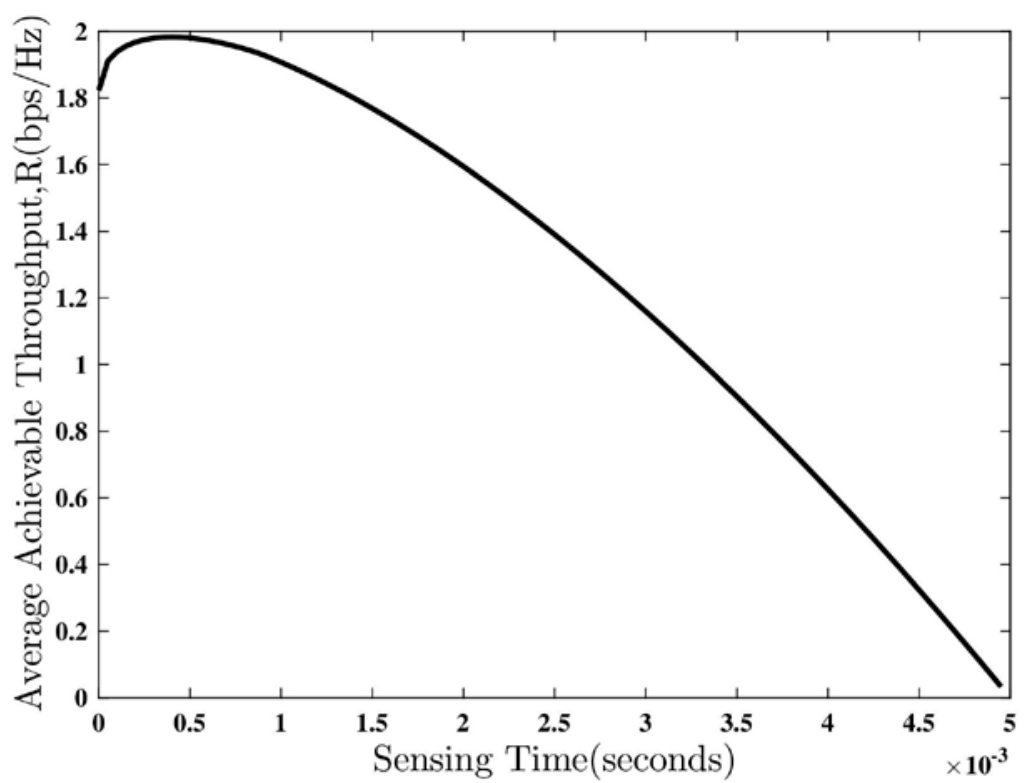

Fig. 2. DF Cognitive relay network spectrum sensing time delay optimization diagram.

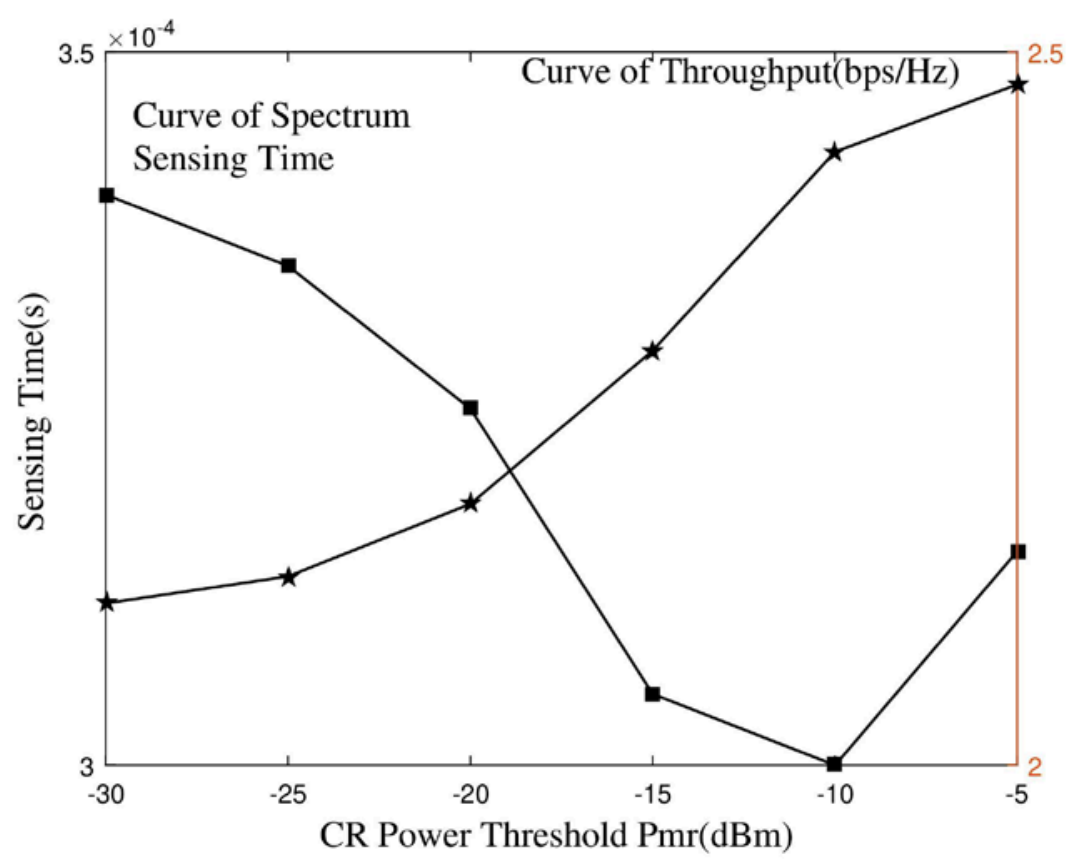

Fig. 3. CR Power Threshold to Spectrum Sensing time Transform diagram. 


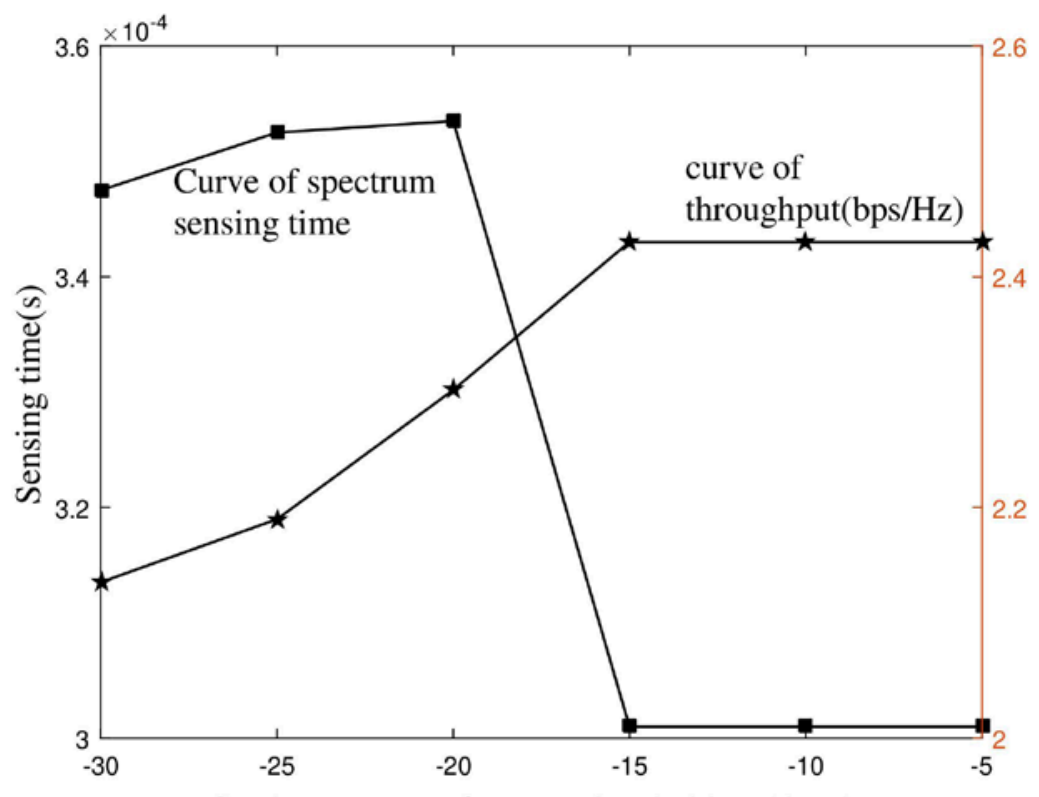

CR Avarage Interference Threshold $\operatorname{Iar}(\mathrm{dBm})$

Fig. 4. CR average interference constraint on the spectrum sensing time transform diagram.

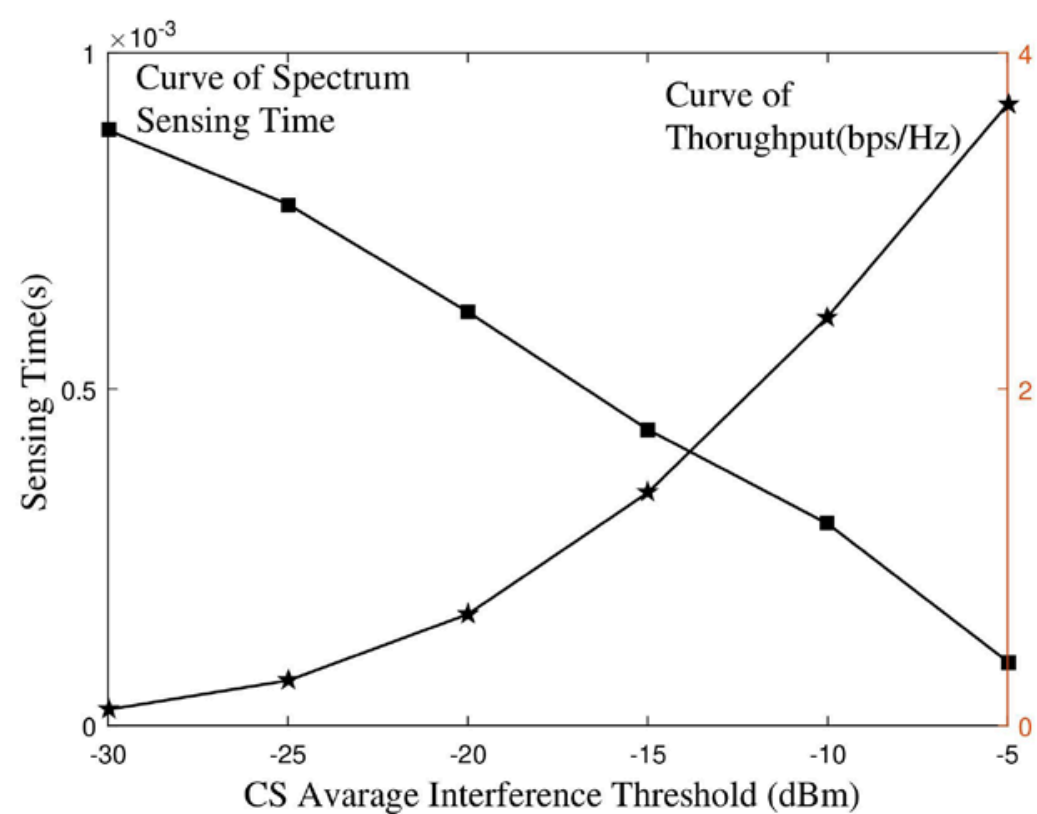

Fig. 5. The time-delay transformation diagram of the spectrum sensing time delay is realized by the average interference constraint of the cognitive relay network.

As shown in Fig. 3, the interchange of the spectrum sensing time and the system throughput can obtain by changing the power threshold of the CR. It can be seen from the figure that as the power threshold increases, the spectrum sensing time shortens and the throughput increases. So allocating more power to the channel can reduce the spectrum sensing delay. 
As shown in Fig. 4, the best interchange trend of the spectrum sensing time and the throughput can obtain by changing the CR average interference constraint $I_{a}{ }^{r}$. From the figure, we can see that the average interference constraint become smaller, the spectrum sensing delay is longer, while the corresponding system throughput decreases.

Fig. 5 shows the interchange trend of the best spectrum sensing time and the system throughput by changing the average interference constraint of the CS. It can be seen from the figure that as the average interference constraint decreases, the spectrum sensing time becomes longer, while the system throughput decreases, which is consistent with the analysis in the previous section.

\section{Conclusion}

The main problem addressed in this paper is to increase the spectrum efficiency of cognitive relay networks. The cognitive radio networks assisted by single-channel cooperative relay adopts the DF mode to improve the spectrum efficiency and increase the communication reliability. The cognitive relay networks using the spectrum sensing mode of listen-first-thenpass, therefore, spent the spectrum sensing time, which affects the throughput and the interference of the channel transmission. The spectrum sensing time for the cognitive relay network can be optimized on the channel interference and the power constraints. The stochastic programming method is used to solve the problem through transforming the uncertainty factors in the constraint into deterministic factors, and an optimal design method of the spectrum sensing channel estimation time is proposed. The computer simulation results show that this method is effective which has certain engineering value.

\section{References}

[1] Mitola Joseph III and G. Q. J. Maguire, “Cognitive radio: making software radios more personal,” IEEE Pers. Commun., vol. 6, no. 4, pp. 13-18, Aug. 1999. Article (CrossRef Link)

[2] E. Astaiza, P. Jojoa, and H. Bermúdez, "Compressive local wideband spectrum sensing algorithm for multiantenna cognitive radios," in Proc. of IEEE 8th Latin-American Conference on Communications (LATINCOM), pp. 1-6, Nov. 15-17, 2016. Article (CrossRef Link)

[3] T. Febrianto, and M. Shikh-Bahaei, "Optimal full-duplex cooperative spectrum sensing in asynchronous cognitive networks," in Proc. of IEEE Asia Pacific Conference on Wireless and Mobile (APWiMob), pp. 1-6, Sept. 13-15, 2016. Article (CrossRef Link)

[4] G. Ozcan, M. C. Gursoy, and et al. "Energy-efficient power allocation in cognitive radio systems with imperfect spectrum sensing," IEEE Journal on Selected Areas in Communications, vol. 34, no. 12, pp. 3466-3481, Dec. 2016. Article (CrossRef Link)

[5] Q. Cui, T. Yuan, and et al, "Energy efficiency analysis of two-way DF relay system with non-ideal power amplifiers," IEEE Communications Letters, vol. 18, no. 7, pp. 1254-1257, July 2014. Article (CrossRef Link)

[6] X. Jiang, L. Shen, and et al, "Power allocation optimisation for high throughput with mixed spectrum access based on interference evaluation strategy in cognitive relay networks," IET Communications, vol. 10, no. 12, pp. 1428-1435, Nov. 2016. Article (CrossRef Link)

[7] A. Sendonaris, E. Erkip, and B. Aazhang, "User cooperation diversity. Part I. System description," IEEE Transactions on Communications, vol. 51, no. 11, pp. 1927-1938, Nov. 2003.

Article (CrossRef Link)

[8] A. Sendonaris, E. Erkip, and B. Aazhang, "User cooperation diversity. Part II. Implementation aspects and performance analysis," IEEE Transactions on Communications, vol. 51, no. 11, pp. 1939-1948, Nov. 2003. Article (CrossRef Link) 
[9] F. Tan, T. Lv, and S. Yang, "Power allocation optimization for energy-efficient massive MIMO aided multi-pair decode-and-forward relay systems," IEEE Transactions on Communications, vol. 65, no. 6, pp. 2368-2381, June 2017. Article (CrossRef Link)

[10] N. Varshney, and A. K. Jagannatham, "Cognitive decode-and-forward MIMO-RF/FSO cooperative relay networks,” IEEE Communications Letters, vol. 21, no. 4, pp. 893-896, April 2017. Article (CrossRef Link)

[11] X. Liu, "A new sensing-throughput tradeoff scheme in cooperative multiband cognitive radio network,” Wiley-Interscience, vol. 24, no. 3, pp. 200-217, May 2014. Article (CrossRef Link)

[12] S. M. Almalfouh, G. L. Stuber, “Joint Spectrum-Sensing Design and Power Control in Cognitive Radio Networks: A Stochastic Approach,” IEEE Trans. on Wireless Commun., vol. 11, no. 12, pp. 4372-4380, Dec. 2012. Article (CrossRef Link)

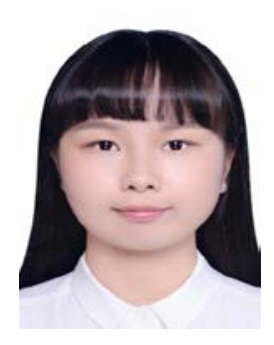

KAILI XIA received her Bachelor's degree in Electronic Information Engineering, China Jiliang University, Hangzhou, China, in 2011. She received her M. Eng. degree in Electronic and Communication Engineering, Hangzhou Dianzi University, Hangzhou, China, in 2018. She is now a software development engineer of Zhejiang Supcon Technology Co., Ltd., Hangzhou, China. Her research interests include cognitive radio networks (CRN), energy efficiency in broadband wireless communications, and Web software development, etc.

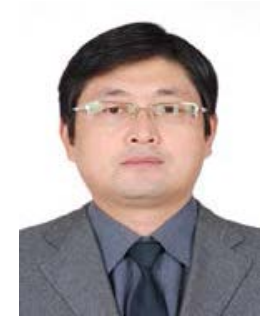

XIANYANG JIANG is with the College of Telecommunication Engineering, Hangzhou Dianzi University (HDU), Hangzhou, China. He received his M. Eng. degree in Communication and Information System from Department of Electronic Engineering, Zhengzhou University, Zhengzhou, China, in 2004. He received his Ph.D. degree major in Communication and Information System from Department of Electronic Engineering, Tsinghua University, Beijing, China, in 2009. Since 2009, he has been with the College of Telecommunication Engineering, HDU. From July 2012 to December 2012, he served as a research scholar with the Electrical and Computer Engineering Department, Stevens Institute of Technology (SIT), Hoboken, NJ, USA. Dr. Jiang's research interests include energy efficiency in broadband wireless communications, cognitive radio networks (CRN), and intelligent transportation, etc.

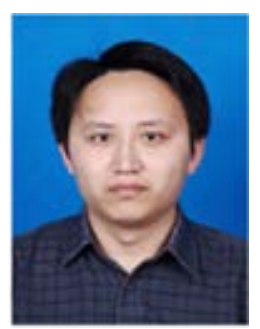

YINGBIAO YAO received Ph.D. degree ininformation and communication engineering from the Zhejiang University, Hangzhou, China, in2006. From 2008 to 2016, he was an Associate Professor with the School of Communication Engineering, Hangzhou Dianzi University, China. In 2011, he was a visiting scholar at RPI University, NY, USA. Since 2017, he has been a Professor with the School of communication engineering, Hangzhou Dianzi University, China. He is the author of three books, more than 50 articles, and more than 30 inventions. His research interests include storage system design, wireless sensor network, indoor localizationand media signal processing. He was arecipient of the third level of Zhejiang Provincial "151" Talent for Excellence in 2015, the first class prize of Zhejiang Provincial Scientific and Technological Progress Award in 2016, and Zhejiang Provincial young and middle-aged academic leaders in 2017.

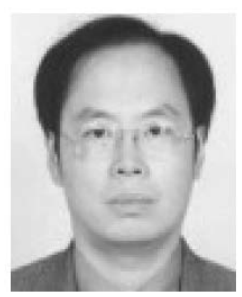

XIANGHONG TANG received the B.S. degree in physics from Southwest Normal University, Chengdu, China, in 1985, the M.S. degree in physics from Sichuan University, Chongqing, in 1988, and the Ph.D.E.E. degree from the University of Electronic Science and Technology, Chengdu, in 1997. He is currently the Dean of the School of Communication Engineering, Hangzhou Dianzi University, Hangzhou, China. His research interests include multimedia signal processing, information theory and source/channel coding. 